\title{
Simulating a one-dimensional plasma
}

\author{
A. D. Boozer ${ }^{\text {a) }}$ \\ Department of Physics, California Institute of Technology, Pasadena, California 91125
}

(Received 20 August 2009; accepted 15 December 2009)

\begin{abstract}
We describe a dynamical model of a one-dimensional plasma and present a simple algorithm for simulating the model on a computer. We use the algorithm to perform several numerical experiments that illustrate collective effects in plasma physics such as Debye screening and plasma oscillations. () 2010 American Association of Physics Teachers.
\end{abstract}

[DOI: $10.1119 / 1.3290442]$

\section{INTRODUCTION}

A plasma is an ionized gas in which some of the electrons are free rather than bound to atoms as in an ordinary gas. The behavior of a plasma is more subtle than the behavior of an ordinary gas. In an ordinary gas the dynamics is dominated by two-body collisions, but in a plasma the particles interact via long-range forces, and this interaction causes the plasma to exhibit collective effects. In this paper we describe a dynamical model of a one-dimensional plasma and present a simple algorithm for simulating the model on a computer. ${ }^{1,2}$ We use computer simulations to illustrate two of the most important collective effects in plasma physics: Debye screening and plasma oscillations. This paper provides an introduction to plasma physics that should be accessible to undergraduates, and can be used to supplement undergraduatelevel courses on plasma physics, statistical mechanics, or numerical modeling.

\section{THE MODEL SYSTEM}

The model system describes $N$ ions confined between two infinitely massive walls separated by a distance $L{ }^{3}$ We assume that the ions collide elastically with the walls but pass freely through one another and interact only via their coupling to the electric field. Let $x_{i}$ and $v_{i}$ denote the position and velocity of ion number $i$, and let $q_{i}$ denote its charge $\left(0 \leq x_{i} \leq L\right)$. For simplicity, we assume that the ions all have the same mass $m$. We take the equations of motion for ion $i$ to be

$$
\dot{x}_{i}=v_{i}, \quad m \dot{v}_{i}=q_{i} E\left(x_{i}\right),
$$

where $E(x)$ is the electric field. The electric field satisfies Gauss's law in one dimension:

$$
\partial_{x} E(x)=2 \rho(x),
$$

where the charge density $\rho(x)$ is given by

$$
\rho(x)=\sum_{i} q_{i} \delta\left(x-x_{i}\right) .
$$

If we substitute Eq. (3) into Eq. (2) and solve for the electric field, we find that

$$
E(x)=\sum_{i} q_{i} \operatorname{sgn}\left(x-x_{i}\right)=\sum_{i} E_{i}(x),
$$

where $E_{i}(x) \equiv q_{i} \operatorname{sgn}\left(x-x_{i}\right)$ is the electric field produced by ion number $i$ and $\operatorname{sgn}(x)$ is the sign function, defined such that $\operatorname{sgn}(x)=1$ for $x>0, \operatorname{sgn}(x)=0$ for $x=0$, and $\operatorname{sgn}(x)=-1$ for $x<0$. Note that $E_{i}(x)$ is the one-dimensional analog of the Coulomb field produced by a point charge in three dimensions. ${ }^{4}$ We can evolve the dynamical variables $x_{i}$ and $v_{i}$ starting from a given initial state by integrating the equations of motion (1) using the expression for the electric field given in Eq. (4) and requiring that the ions collide elastically with the walls.

It is convenient to introduce a scalar potential $\phi(x)$ such that $E(x)=-\partial_{x} \phi(x)$. From Eq. (4) we find that

$$
\phi(x)=-\sum_{i} q_{i}\left|x-x_{i}\right| .
$$

The potential energy of the system is

$$
V=\frac{1}{2} \sum_{i} q_{i} \phi\left(x_{i}\right)=-\frac{1}{2} \sum_{i j} q_{i} q_{j}\left|x_{i}-x_{j}\right| .
$$

The kinetic energy is

$$
K=\frac{m}{2} \sum_{i} v_{i}^{2} .
$$

Using the equations of motion (1), one can show that the total energy $U=K+V$ is a constant of the motion.

So far we have assumed that the ions have arbitrary charges $q_{i}$, but in what follows we will focus on the special case of a two-component plasma in which half of the ions have charge $e$ and the other half have charge $-e$. We define $c_{i} \equiv q_{i} / e$ to be the sign of the charge of ion number $i$.

\section{COLLECTIVE EFFECTS}

We now briefly review two important collective effects that plasmas can exhibit: Debye screening and plasma oscillations.

If a positive test charge is placed inside a plasma, it will attract negative ions and repel positive ions, thereby creating a cloud of ions around itself of net negative charge. The presence of this cloud screens the test charge and modifies its electric field. This phenomena is known as Debye screening. ${ }^{5-7}$

Let us consider Debye screening in the context of our one-dimensional plasma model. We assume that the plasma is in thermal equilibrium at temperature $T$. Let $n_{+}(x)$ and $n_{-}(x)$ denote the number densities of positive and negative ions at position $x$, and let $\bar{n}$ denote the spatial average of these quantities across the entire length of the plasma. (Because there are equal numbers of positive and negative ions, the spatial averages of $n_{+}(x)$ and $n_{-}(x)$ must be equal.) Suppose we place a test charge $Q$ at position $x_{0}$. The charge density is then given by 


$$
\rho(x)=e n_{+}(x)-e n_{-}(x)+Q \delta\left(x-x_{0}\right) .
$$

An ion with charge $\pm e$ at position $x$ has potential energy $\pm e \phi(x)$, so the number density $n_{ \pm}(x)$ is given by the mean number density $\bar{n}$ weighted by the Boltzmann factor $\exp (\mp \beta e \phi(x))$, where $\beta=1 / k_{B} T$ is the inverse temperature:

$$
n_{ \pm}(x)=\bar{n} e^{\bar{\mp} \beta \phi(x)} .
$$

We will assume that $\beta e \phi(x) \ll 1$, so we can approximate Eq. (9) by

$$
n_{ \pm}(x) \simeq \bar{n}[1 \mp \beta e \phi(x)] .
$$

The scalar potential $\phi(x)$ satisfies Poisson's equation,

$$
\partial_{x}^{2} \phi(x)=-\partial_{x} E(x)=-2 \rho(x) .
$$

From Eqs. (8), (10), and (11), we find that

$$
\left(\partial_{x}^{2}-k_{D}^{2}\right) \phi(x)=-2 Q \delta\left(x-x_{0}\right),
$$

where $k_{D}^{2} \equiv 4 \beta e^{2} \bar{n}$. The solution to Eq. (12) is

$$
\phi(x)=\left(Q / k_{D}\right) e^{-k_{D}\left|x-x_{0}\right|} .
$$

The corresponding electric field is

$$
E(x)=-\partial_{x} \phi(x)=Q \operatorname{sgn}\left(x-x_{0}\right) e^{-k_{D}\left|x-x_{0}\right|} .
$$

In free space the electric field of the test charge would be $E(x)=Q \operatorname{sgn}\left(x-x_{0}\right)$. The presence of the plasma modifies this field, causing it to fall off exponentially with distance. The fall-off is characterized by a length scale $\lambda_{D} \equiv 1 / k_{D}$ $=\left(4 \beta e^{2} \bar{n}\right)^{-1 / 2}$ called the Debye length.

Let us define functions

$$
p_{ \pm}(x)=\left[n_{+}(x)+n_{-}(x)\right]^{-1} n_{ \pm}(x)
$$

that give the probability for an ion located at position $x$ to have charge $\pm e$. From Eqs. (10) and (13), we find that

$$
p_{ \pm}(x)=(1 / 2)\left(1 \mp \alpha e^{-k_{D}\left|x-x_{0}\right|}\right),
$$

where $\alpha \equiv \beta e Q / k_{D}{ }^{8}$ Equation (16) tells us that far away from the test charge we are equally likely to find an ion of either charge, but near the test charge we are more likely to find an ion of the opposite charge than an ion of the same charge. Note that although we have described Debye screening for the case of an externally imposed test charge, the ions themselves can also be viewed as test charges. Thus, each ion is surrounded by an oppositely charged cloud of size $\lambda_{D}$.

As another example of a collective effect, we consider the phenomena of plasma oscillations. ${ }^{9-11}$ If we displace the positive ions with respect to the negative ions, the mutual attraction of the two ion species acts as a restoring force that causes them to oscillate relative to one another. We can describe the oscillations as follows.

Suppose we displace all the positive ions by $d / 2$ and all the negative ions by $-d / 2$. The displacements create two charged regions at either end of the plasma: one that consists of positive ions and has net charge $+e \bar{n} d$, and one that consists of negative ions and has net charge $-e \bar{n} d$. The charged regions produce a uniform electric field $E=-2 e \bar{n} d$ in the bulk of the plasma. This field exerts a force $e E$ on the positive ions and a force $-e E$ on the negative ions, so the relative acceleration of the two ion species is

$$
\ddot{d}=2 e E / m=-\omega_{p}^{2} d,
$$

where $\omega_{p} \equiv\left(4 e^{2} \bar{n} / m\right)^{1 / 2}$ is called the plasma frequency. ${ }^{12}$ Equation (17) tells us that the two ion species oscillate relative to one another at the plasma frequency.

\section{NUMERICAL EXPERIMENTS}

We will now illustrate these collective effects by simulating the model on a computer and performing several numerical experiments. It will be convenient to choose a system of units in which distance is measured in units of $L$, time is measured in units of $\left(m L / e^{2}\right)^{1 / 2}$, and mass is measured in units of $m$. Such a choice of units renders all quantities dimensionless.

For each experiment we choose an initial state and evolve it in time by integrating the equations of motion. The algorithm used to perform the integration is described in the Appendix. For these experiments we use $N=100$ ions.

To obtain an initial state with total energy $U_{0}$, we use the following algorithm. First we set $c_{i}=1$ for $i \leq N / 2$ and $c_{i}=-1$ for $i>N / 2$. Assume we have a random number generator that produces numbers uniformly distributed from zero to one. We use the generator to choose $N$ random numbers $r_{1}, \ldots, r_{N}$, and we set the ion positions to $x_{i}=r_{i}$. We then use Eq. (6) to compute the potential energy $V_{0}$. If $V_{0}>U_{0}$, we choose another set of random numbers and try again; we keep trying until we obtain a set of ion positions for which $V_{0} \leq U_{0}$.

Now we are ready to choose the ion velocities. Because we want the total energy to be $U_{0}$ and the potential energy is $V_{0}$, we must choose the velocities such that the root-meansquared velocity $\widetilde{v}_{0}$ satisfies

$$
(\mathrm{Nm} / 2) \widetilde{v}_{0}^{2}=U_{0}-V_{0} .
$$

We satisfy this requirement as follows. We choose $N$ random numbers $r_{1}, \ldots, r_{N}$, define quantities $w_{i} \equiv 2 r_{i}-1$, which are uniformly distributed from -1 to 1 , and compute their rootmean-square $\widetilde{w}$. We then define $v_{i}=\eta w_{i}$, where the constant $\eta \equiv \widetilde{v}_{0} / \widetilde{w}$ is chosen such that the root-mean-square velocity is $\widetilde{v}_{0}$. This last step completes the choice of the initial state.

This algorithm produces initial states in which the total energy is $U_{0}$ and the ion velocities are uniformly distributed from $-\sqrt{3} \widetilde{v}_{0}$ to $\sqrt{3} \widetilde{v}_{0} \cdot{ }^{13}$ Because the distribution of ion velocities is non-Maxwellian, for these initial states the plasma is not in thermal equilibrium.

For our first experiment, we simulate the evolution of the system toward thermal equilibrium starting from these nonequilibrium initial states. We choose 100 initial states, each with total energy $U_{0}=10.0$. We evolve each initial state and compute the kinetic energy $K$ as a function of time; we then average $K(t)$ over all the initial states to obtain the average kinetic energy $\bar{K}(t)$.

In Fig. 1 we plot $\bar{K}(t)$ versus time. We see that the average kinetic energy starts at $\bar{K}_{0}=3.53$ and evolves to the equilibrium value $\bar{K}_{E}=8.53$. The reason the kinetic energy increases is that the ion positions are initially uncorrelated, ${ }^{14}$ but in equilibrium each ion is surrounded by a cloud of oppositely charged ions. The formation of these clouds lowers the potential energy, thus raising the kinetic energy.

This experiment provides a simple way of obtaining equilibrium states: to obtain an equilibrium state with total en- 


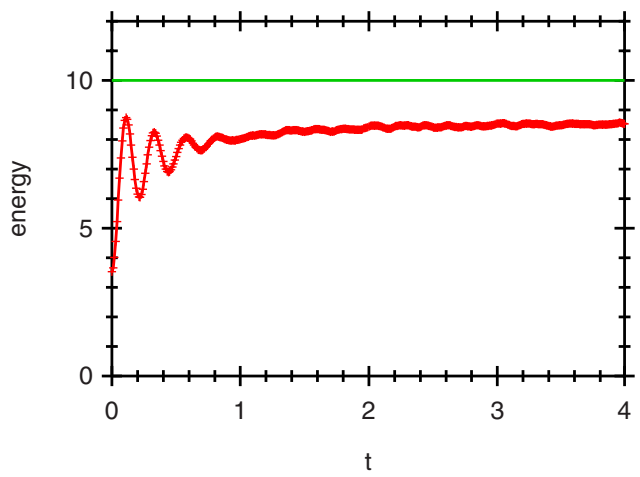

Fig. 1. Evolution toward thermal equilibrium. The points give the average kinetic energy $\bar{K}(t)$ versus the time $t$; the solid line is the total energy $U_{0}=10.0$.

ergy $U_{0}$, we choose a nonequilibrium state using the algorithm we have described and evolve it in time by $\Delta t=4 .{ }^{15} \mathrm{We}$ can determine the temperature $T$ of the resulting equilibrium states by using the equipartition theorem, which states that

$$
k_{B} T / 2=(m / 2) \widetilde{v}^{2}=\bar{K}_{E} / N,
$$

where $\widetilde{v}$ is the equilibrium root-mean-squared velocity. We substitute for $\bar{K}_{E}$ and find that the temperature is $k_{B} T$ $=0.171$ and the equilibrium root-mean-squared velocity is $\widetilde{v}=0.413$.

For our second experiment we check that the distribution of ion velocities is Maxwellian when the system is in thermal equilibrium. We use the method we have described to choose 100 equilibrium states, each with total energy $U_{0}=10.0$, and we calculate the average distribution of ion velocities. ${ }^{16}$ In Fig. 2 we plot the resulting velocity distribution together with the Maxwell distribution

$$
f_{M}(v)=\left(2 \pi \tilde{v}^{2}\right)^{-1 / 2} e^{-v^{2} / 2 \tilde{v}^{2}} .
$$

We find good agreement between the numerical and theoretical distributions.

For our third experiment we illustrate Debye screening. We first choose $10^{3}$ equilibrium states, each with total energy $U=10.0$. For each state we compute the probability $P_{+}(x)$ that two ions separated by a distance $x$ have the same charge, and the probability $P_{-}(x)$ that they have opposite charges. We average $P_{+}(x)$ and $P_{-}(x)$ over all the states to obtain



Fig. 2. Distribution of ion velocities. The jagged curve is the numerically determined velocity distribution; the smooth curve is the Maxwell distribution.



Fig. 3. Debye screening. The points give the probabilities that a pair of ions separated by a distance $x$ has the same charge $\left(P_{+}(x)\right)$ or opposite charges $\left(P_{-}(x)\right)$; the smooth curves are the corresponding theoretical predictions $p_{+}(x)$ and $p_{-}(x)$.

$\bar{P}_{+}(x)$ and $\bar{P}_{-}(x)$. Based on the results in Sec. III, we expect that $\bar{P}_{ \pm}(x)=p_{ \pm}(x)$, where $p_{ \pm}(x)$ is given by Eq. (16) with $x_{0}=0$ and $Q=e$.

In Fig. 3 we plot the numerically determined probabilities $\bar{P}_{ \pm}(x)$ and the theoretical predictions $p_{ \pm}(x)$ and find good agreement. ${ }^{17}$ The parameters $k_{D}=34.2$ and $\alpha=0.171$ in Eq. (16) for $p_{ \pm}(x)$ are calculated using the temperature $T$ that we obtained from Eq. (19). Figure 3 shows that each ion is surrounded by an oppositely charged cloud of size $\lambda_{D}=1 / k_{D}$ $=0.0292$, which is about three times the mean interparticle spacing.

For our fourth experiment we illustrate plasma oscillations. We first choose 100 equilibrium states, each with total energy $U=10.0$. For each initial state we give the positive ions a positive momentum kick and the negative ions a negative momentum kick; that is, we make the replacements $v_{i}$ $\rightarrow v_{i}+c_{i} A / 2$, where $A=0.2$. We define $V$ to be the average velocity difference between positive and negative ions:

$$
V=\frac{2}{N} \sum_{i} c_{i} v_{i}
$$

We evolve each initial state and compute $V$ as a function of time; we then average $V(t)$ over all the initial states to obtain the average velocity difference $\bar{V}(t)$.

In Fig. 4 we plot $\bar{V}(t)$ and the function



Fig. 4. Plasma oscillations. The points give the average velocity difference $\bar{V}(t)$ versus the time $t$; the smooth curve is the damped sinusoid given in Eq. (22). 


$$
f(t)=A e^{-t / \tau} \cos \omega_{p} t,
$$

where the parameter $\tau=0.84$ is obtained by fitting $f(t)$ to the numerical data. We observe oscillations at the plasma frequency $\omega_{p}=(2 \pi)(2.25)$, as predicted in Sec. III. The oscillations are damped, as can be understood from the following considerations. The momentum kick throws the system out of thermal equilibrium, because after the kick the velocity distribution of the ions is no longer Maxwellian. As the system evolves back to equilibrium, the velocity distribution returns to the Maxwell distribution and the average velocity difference $V$ goes to zero. Thus, the oscillations decay over a timescale $\tau$ that characterizes the time it takes the system to re-equilibrate.

\section{CONCLUSION}

We have presented a simple model of a one-dimensional plasma, described how to simulate the model on a computer, and used the simulation to illustrate Debye screening and plasma oscillations. The simulation is based on an algorithm that relies on the unique features of one-dimensional plasmas and cannot be generalized to higher dimensions. The collective effects, however, do carry over naturally to threedimensional plasmas. ${ }^{18}$ For a three-dimensional plasma, a test charge $Q$ at the origin generates an electric field

$$
\vec{E}(\vec{r})=Q e^{-r / \lambda_{D}} \hat{r} / r^{2},
$$

where $\lambda_{D} \equiv\left(8 \pi \beta e^{2} \bar{n}\right)^{-1 / 2}$ is the Debye length. Thus, as for a one-dimensional plasma, the electric field of a test charge is exponentially suppressed over a characteristic length $\lambda_{D}$. A three-dimensional plasma can also exhibit plasma oscillations, with the plasma frequency given by $\omega_{p}=(8 \pi \bar{n} / m)^{1 / 2}$.

As an additional application of the model presented here and a possible project for students, we suggest that it would be interesting to investigate the behavior of the system when the sign of the force law is flipped so that like charges attract and opposite charges repel. The sign flip can be accomplished by replacing the equations of motion (1) with

$$
\dot{x}_{i}=v_{i}, \quad m \dot{v}_{i}=-q_{i} E\left(x_{i}\right) .
$$

In particular, the sign-flipped model could be used to study the statistical mechanics of gravitationally interacting particles. The statistical mechanics of such systems is relevant to important issues in cosmology, such as the gravitational clumping of matter in the early universe. ${ }^{19}$

\section{APPENDIX: SIMULATION ALGORITHM}

Here we describe an algorithm for efficiently simulating the model system on a computer. Assume we know the state of the system at time $t_{0}$. Let us order the ions according to their position so that $x_{1}<x_{2}<\ldots<x_{N}$. Define integers $a_{i}$ by

$$
a_{i}=c_{i} \sum_{j<i} c_{j}-c_{i} \sum_{j>i} c_{j} .
$$

From Eqs. (1) and (4), we see that the integer $a_{i}$ describes the acceleration experienced by ion number $i$ due to its coupling to the electric field.

If we evolve the initial state, we will eventually reach a time $t_{1}=t_{0}+\tau$ at which one of three events occurs: (1) a pair of ions $i$ and $i+1$ meet so that $x_{i}=x_{i+1},(2)$ ion 1 collides with the left wall so that $x_{1}=0$, or (3) ion $N$ collides with the right wall so that $x_{N}=1$. We calculate $\tau$ by determining which of these events occurs first. For events of type 1, we define times $\tau_{i}$ for $i=1, \ldots, N-1$ by

$$
x_{i+1}-x_{i}+\left(v_{i+1}-v_{i}\right) \tau_{i}+\left(a_{i+1}-a_{i}\right) \tau_{i}^{2} / 2=0 .
$$

If Eq. (A2) has two positive solutions, we define $\tau_{i}$ to be the smaller solution, and if it has no positive solutions, we assign $\tau$ a value $\tau_{\max }$ that is sufficiently large to eliminate the corresponding event as a candidate for the first event. Similarly, for events of types 2 and 3 we define times $\tau_{L}$ and $\tau_{R}$ by

$$
\begin{aligned}
& x_{1}+v_{1} \tau_{L}+a_{1} \tau_{L}^{2} / 2=0, \\
& x_{N}+v_{N} \tau_{R}+a_{N} \tau_{R}^{2} / 2=1 .
\end{aligned}
$$

To obtain $\tau$ we take the minimum of $\tau_{1}, \ldots, \tau_{N-1}, \tau_{R}, \tau_{L}$. If $\tau=\tau_{i}$, ion $i$ meets ion $i+1$; if $\tau=\tau_{L}$, ion 1 collides with the left wall; and if $\tau=\tau_{R}$, ion $N$ collides with the right wall.

We are now ready to evolve the system from $t_{0}$ to just before $t_{1}$. For times $t_{0} \leq t<t_{1}$ we can express the equations of motion (1) as

$$
\dot{x}_{i}=v_{i}, \quad \dot{v}_{i}=a_{i} .
$$

Thus, to evolve the system from $t_{0}$ to just before $t_{1}$, we make the replacements

$$
x_{i} \rightarrow x_{i}+v_{i} \tau+a_{i} \tau^{2} / 2, \quad v_{i} \rightarrow v_{i}+a_{i} \tau .
$$

We evolve the system from just before $t_{1}$ to just after $t_{1}$ as follows. If ion $i$ meets ion $i+1$, we swap the two ions by making the replacements

$$
\left(\begin{array}{c}
x_{i} \\
v_{i} \\
c_{i} \\
a_{i}
\end{array}\right) \rightarrow\left(\begin{array}{c}
x_{i+1} \\
v_{i+1} \\
c_{i+1} \\
a_{i+1}^{\prime}
\end{array}\right), \quad\left(\begin{array}{c}
x_{i+1} \\
v_{i+1} \\
c_{i+1} \\
a_{i+1}
\end{array}\right) \rightarrow\left(\begin{array}{c}
x_{i} \\
v_{i} \\
c_{i} \\
a_{i}^{\prime}
\end{array}\right),
$$

where $a_{i}^{\prime}=a_{i}+2 c_{i} c_{i+1}$ and $a_{i+1}^{\prime}=a_{i+1}-2 c_{i} c_{i+1}$. If ion 1 collides with the left wall, we make the replacement $v_{1} \rightarrow-v_{1}$. If ion $N$ collides with the right wall, we make the replacement $v_{N}$ $\rightarrow-v_{N}$. We have now evolved the system from time $t_{0}$ to just after time $t_{1}$. By iterating this procedure, we can evolve the system indefinitely.

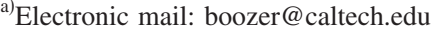

${ }^{1}$ A similar model of a one-dimensional plasma is described in J. M. Dawson, "One-dimensional plasma model," Phys. Fluids 5, 445-459 (1962), and J. Dawson, "Thermal relaxation in a one-species, one-dimensional plasma," ibid. 7, 419-425 (1964). Dawson's model describes a onespecies plasma with a uniform neutralizing background charge. In contrast, the model considered here describes a two-species plasma with no background charge.

${ }^{2}$ The computer program used to perform the simulation is available upon request.

${ }^{3}$ As we describe in Sec. III, the plasma is characterized by a correlation length $\lambda_{D}$ called the Debye length. Ions within $\lambda_{D}$ of the walls may have somewhat different properties from ions further away from the walls. Because we will only consider situations in which $\lambda_{D} \ll L$, these differences are unimportant for our purposes.

${ }^{4}$ Note that unlike the Coulomb field, the field $E_{i}(x)$ does not fall off with distance. As shown in the Appendix, this property greatly simplifies the task of simulating the system on a computer.

${ }^{5}$ Debye screening was first discussed in P. Debye and E. Hückel, "Zur theorie der elektrolyte," Phys. Z. 9, 184-206 (1923).

${ }^{6} \mathrm{An}$ introduction to Debye screening is given in R. P. Feynman, R. B. Leighton, and M. Sands, The Feynman Lectures on Physics (AddisonWesley, Reading, MA, 1963), Vol. 2, Sec. 7-4.
} 
${ }^{7}$ See also N. Meyer-Vernet, "Aspects of Debye shielding," Am. J. Phys. 61 (3), 249-257 (1993).

${ }^{8}$ Note that the approximation $\beta e \phi(x) \ll 1$ is self-consistent provided that $\alpha \ll 1$.

${ }^{9}$ Plasma oscillations were first discussed in L. Tonks and I. Langmuir, "Oscillations in ionized gases," Phys. Rev. 33, 195-210 (1929).

${ }^{10} \mathrm{An}$ introduction to plasma oscillations is given in Ref. 6, Sec. 7-3.

${ }^{11}$ See also J. D. Jackson, Classical Electrodynamics, 2nd ed. (Wiley, New York, 1975), Sec. 10.8.

${ }^{12}$ Note that the plasma frequency is independent of temperature.

${ }^{13}$ Note that the value of $\widetilde{v}_{0}$ will vary from initial state to initial state.

${ }^{14}$ The ion positions for the initial state are not completely uncorrelated because our state selection algorithm rejects states for which $V_{0}>U_{0}$.
${ }^{15}$ As shown in Fig. 1, this time interval is sufficiently long to allow the state to reach thermal equilibrium.

${ }^{16} \mathrm{We}$ compute the velocity distribution by making a histogram of the ion velocities $v_{i}$. The bin width for the histogram is $\Delta v=0.05$.

${ }^{17}$ We compute the probabilities $P_{ \pm}(x)$ by making histograms of the ion separations $\left|x_{i}-x_{j}\right|$. The bin width for these histograms is $\Delta x=0.004$.

${ }^{18} \mathrm{~A}$ numerical calculation of the mean electrostatic potential energy for a three-dimensional plasma is discussed in O. Theimer and M. M. Theimer, "Computer simulation of statistical plasma mechanics (an example of undergraduate research)," Am. J. Phys. 46 (6), 661-666 (1978).

${ }^{19}$ See, for example, T. Rothman and P. Anninos, "Phase space approach to the gravitational arrow of time," Phys. Rev. D 55 (4), 1948-1963 (1997), and references therein.

\title{
APS/AAPT Online Journal: Physical Review Special Topics - Physics Education Research
}

A peer-reviewed, electronic journal exploring experimental and theoretical research on the teaching and learning of physics.

\author{
Edited by: \\ Robert Beichner \\ North Carolina State University
}

Access Is Free!

\section{Features:}

- review articles

- methodology critiques

- new assessment tools and research techniques

To learn more about the PER journal visit: http://www.aapt.org/Publications/perjournal.cfm

Physical Review Special Topics-Physics Education Research is sponsored by APS, AAPT, and the APS Forum on Education. 